\title{
Z-1 Prototype Space Suit Testing Summary
}

The Advanced Space Suit team of the NASA-Johnson Space Center performed a series of test with the Z1 prototype space suit in 2012 . This paper discusses, at a summary level, the tests performed and results from those tests. The purpose of the tests were two-fold: 1) characterize the suit performance so that the data could be used in the downselection of components for the Z-2 Space Suit and 2) develop interfaces with the suitport and exploration vehicles through pressurized suit evaluations. Tests performed included isolated and functional range of motion data capture, Z-1 waist and hip testing, joint torque testing, $\mathrm{CO}_{2}$ washout testing, fit checks and subject familiarizations, an exploration vehicle aft deck and suitport controls interface evaluation, delta pressure suitport tests including pressurized suit don and doff, and gross mobility and suitport ingress and egress demonstrations in reduced gravity. Lessons learned specific to the Z-1 prototype and to suit testing techniques will be presented.

Authored by: Amy Ross/EC5

Abstract for submission to the $43^{\text {rd }}$ International Conference on Environmental Systems 